\title{
EVALUATION OF ENVIRONMENTAL NEUTRON DOSE AT GROUND LEVEL
}

\author{
N. Marchese, A. Parlato, E.A.G. Tomarchio*
}

Department of Engineering, University of Palermo, Palermo, Italy

\begin{abstract}
This work presents the results of two cycles of neutron dose rate measurements realized using an ALNOR 2202D Neutron Dose Rate Meter whose time response is acquired and analyzed through a controlled ORTEC MCS-32 acquisition card in Windows environment. The data obtained have been compared with values from previous experimental surveys and with the data provided by the worldwide main observatories. It has been also verified the influence of the fluctuations in the flux of cosmic rays during the course of a solar cycle. By comparing the realized measurements and the data provided by the cosmic ray monitoring networks it is also possible to obtain a value of ambient dose equivalent rate and neutron flux rate which can be used as a reference for design a neutron irradiation testing of electronic devices.
\end{abstract}

Keywords: Environmental neutron dose, rem Counter, neutron flux, solar radiation

\section{INTRODUCTION}

A significant interest has been shown recently on the evaluation of the neutron flux at ground level to determine its impact on specific instrumentation and devices (from complex telecommunications systems to home electronic devices appliances).

The present study deals with the analysis of the neutron flux due to the interaction of cosmic rays (both the galactic and solar ones) with the outer layers of the Earth atmosphere.

The neutron field can be influenced by fluctuations on the primary flux of cosmic rays mainly due to phenomena related to solar activity cycles. The reference solar cycle has a period of approximately 11 years (Schwabe cycle), during which solar activity varies from minimum values (start and end of the cycle) to the maximum value, approximately in the middle of the cycle itself. This variation influences the flux of cosmic rays incident on Earth and, then, the atmospheric neutron field.

The regularity of the time evolution of solar activity is sometimes interrupted by periods of great depression of the activity called large "minima", but also by solar events (flares and coronal emissions), solar modulation, interaction with the Earth's magnetosphere, and others of minor importance.

Solar modulation greatly affects the intensity of cosmic rays, as the solar activity increases, the flux of cosmic rays decreases and vice versa. This inverse correlation is confirmed by the analysis of the ground level concentrations of radionuclides produced in the atmosphere by spallation of cosmic rays, such as $7 \mathrm{Be}$, which has a characteristic modulation both in the short term, seasonal, and long term with a time around 11 years. This modulation was highlighted in [1-3] with reference to $7 \mathrm{Be}$ air concentration measurements during two decades of observations.

In many applications it is important to know the neutron dose rate at ground level, to assess any effects on materials and devices, and ultimately also on humans, in terms of external neutron exposure. Furthermore, to design functioning testing for various electronic devices, it is very important to be able to evaluate quickly the neutron dose value at ground level with commonly used instrumentation.

For this purpose, the results of two measurement campaigns in the periods February-March 2012 and June-July 2020 are shown. The periods chosen fall approximately in a maximum and minimum phase of solar activity. The data obtained through the use of a common rem-meter allow to evaluate an average neutron dose rate, in terms of ambient dose equivalent rate, $\dot{H}^{*}(10)$. This allows to determine the parameters of neutron irradiation testing to study the sensitivity of various devices to neutron exposure and to evaluate a correlation with solar activity.

A validation of the procedure has been obtained by comparing the results of measurements with the ones carried out in other locations, finding a good agreement between measurements realized with different conditions.

\footnotetext{
*elio.tomarchio@unipa.it
} 
In addition, a procedure for determining a corrective factor to take into account the variations in the flux of cosmic rays during a solar cycle has been assessed using the data from the Neutron Monitor Data Base (NMDB), a global monitoring network that records the trend of the flux of cosmic rays that affect the Earth's atmosphere [4].

\section{MATERIALS AND METHODS}

Dose rate measurements have been performed using an ALNOR 2202D neutron dose rate meter consisting of a cylindrical $\mathrm{BF}_{3}$ proportional counter with high sensitivity to neutrons, surrounded by a shield consisting of polyethylene and borated polyethylene to obtain a response similar to that of human biological tissue (Fig. 1). The use of the $\mathrm{BF}_{3}$ proportional counter for neutron detection offers the advantage of efficient discrimination from the background gamma radiation associated with neutron fields.

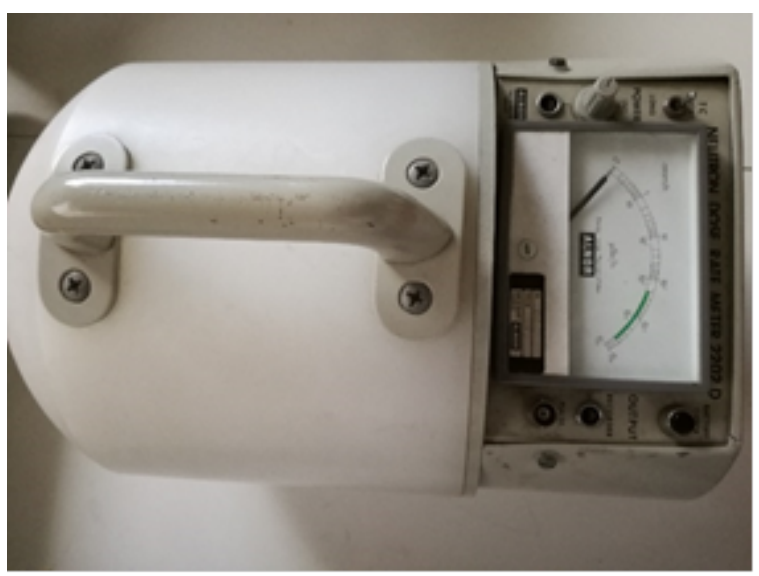

Figure 1. Photograph of ALNOR 2202D Rem-meter.

Since the value of the environmental neutron dose rate is lower than the minimum value of the analog measuring scale on the instrument, a pulse counter has been set up directly coupled with the $\mathrm{BF}_{3}$ detector and connected to a remote count analysis system based on a Multiscaler implemented on an ORTEC MCS-32 card in Windows environment. The software allows to set up the parameters of the board for acquiring data from the detector through specific job files. The series of measurements have been carried out almost continuously in the time periods 21 February - 7 March 2012 and 20 June - 4 July 2020 with the rem-meter positioned inside a pre-assembled sheet metal structure placed on the roof of the department (about $20 \mathrm{~m}$ above street level) normally used for housing equipment for environmental measurements [1,2].

To allow a long-time use of the instrumentation, a special cable has been realized to connect the meter to an external continuous power supply system instead of the battery power supply. This also made possible to remotely transfer the information in real time to the acquisition station to be stored for post processing. The counting has been extended over 6 hours to obtain precision in results below $10 \%$. The $H^{*}(10)$ value has been calculated through the use of the relation

$$
\dot{H}^{*}(10)=\mathrm{M} \times \mathrm{C} \times \mathrm{T}
$$

where $M$ (cps, counts per second) is the measurement result, $\mathrm{C}$ is the conversion coefficient of $3.03 \mu \mathrm{Sv} \mathrm{h}{ }^{-1}$ $\mathrm{cps}^{-1}$ given by the manufacturer, $\mathrm{T}$ is a calibration factor provided for the two time periods by the National Institute of Ionizing Radiation Metrology (INMRI, located at the ENEA Casaccia Research Centre, near Rome, Italy) and by Comecer Calibration Center (located at Castel Bolognese, Ravenna, Italy), respectively.

\section{RESULTS AND DiSCUSSION}

The results of the measurements are shown in Figs. 2 and 3 and compared with the trend of the Sunspot number in the same time periods obtained from [5].

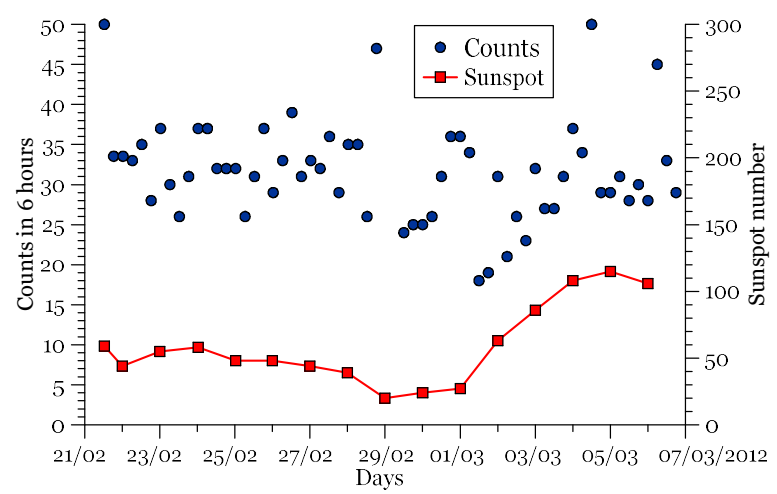

Figure 2. Qualitative comparison between the data measured with the ALNOR 2202 D rem-meter and the sunspot number in the period 21 February - 7 March 2012.

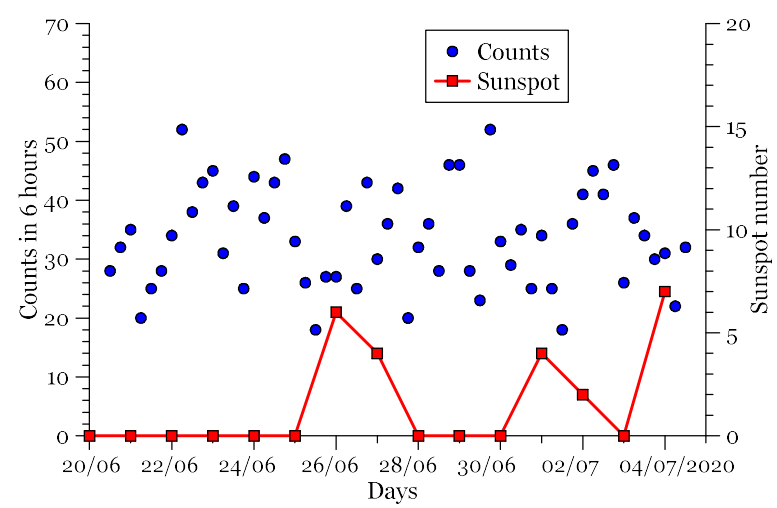

Figure 3. Qualitative comparison between the data measured with the ALNOR 2202D rem-meter and the sunspot number in the period 20 June -4 July 2020.

The results are summarized for the two time periods in Tab. 1 where, to have a feedback on the quality of the measurement method, a comparison with data reported by Nunomiya et al. [6], Nakamura et al. [7-9], Kowatari et al. [10] and Lee et al. [11] is performed, even if referred to different time periods. 
It can be noted that the neutron flux rate values obtained in both time periods are lower to the ones found in other locations. This can be due to the reduced range of energies for which the ALNOR rem meter is sensitive (up to $17 \mathrm{MeV}$ ). However, the $\dot{H}^{*}(10)$ value of $4.5 \mathrm{nSv} \mathrm{h}{ }^{-1}$, considered $8760 \mathrm{~h} /$ year of exposure, leads to an annual dose value of $39 \mu \mathrm{Sv} / \mathrm{year}$, close to the values $30.5 \mu \mathrm{Sv} /$ year by [11] and $31 \mu \mathrm{Sv} /$ year by [12].

Table 1. Comparison of the measured values in different locations and periods

\begin{tabular}{|l|c|c|}
\hline \multicolumn{1}{|c|}{ Quantity } & $\begin{array}{c}\text { Neutron flux } \\
\text { rate } \\
\left(\times 10^{-3} \mathrm{~cm}^{-2} \mathrm{~s}^{-1}\right)\end{array}$ & $\begin{array}{c}\text { Ambient Dose } \\
\text { Equivalent rate } \\
\left(\mathrm{nSv} \mathrm{h}^{-1}\right)\end{array}$ \\
\hline This work & $\begin{array}{l}\text { 1) } 4.6 \pm 0.2^{(4)} \\
\text { 2) } 4.8 \pm 0.2^{(4)}\end{array}$ & $\begin{array}{l}4.5 \pm 0.2 \\
4.7 \pm 0.2\end{array}$ \\
\hline $\begin{array}{l}\text { Numomiya et al., } \\
\text { 2004 [6] }{ }^{(1)}\end{array}$ & 5.4 & $4.1 \pm 5 \%$ \\
\hline $\begin{array}{l}\text { Nakamura et al., } \\
\text { 2005 [8] }{ }^{(1)}\end{array}$ & 5.4 & $4.0 \pm 0.2$ \\
\hline $\begin{array}{l}\text { Kowatari et al., } \\
\text { 2007 [10] }{ }^{(2)}\end{array}$ & $6.3 \pm 0.26$ & $5.4 \pm 0.23$ \\
\hline $\begin{array}{l}\text { Lee et al., 2017 } \\
\text { [11] (3) }\end{array}$ & 5.39 & \\
\hline
\end{tabular}

(1) Sendai, Japan, April 2001-March 2003, neutron flux rate calculated for energies up to $20 \mathrm{MeV}$.

(2) Kagoshima, Japan, November 2003, neutron flux rate estimated from the measured neutron energy spectrum. (3) Hsinchu, Taiwan, February 2015, neutron flux rate calculated for the whole neutron energy spectrum.

(4) Neutron energy range up to $17 \mathrm{MeV}$.

Measurements of the neutron flux rate carried out over a short time period do not allow an assessment of its dependence on primary cosmic rays variation during a solar cycle. To verify the influence of solar cycle on the average neutron fluence rate in the atmosphere, an approach similar to the one reported in [13] has been applied. The method is based on the comparison of measurements and data from NMDB network stations [4] with solar activity data. In particular, with reference to stations closest to the measurement site, the variation on the measurement of neutron flux (or other correlated parameter, e.g. cps) with respect of an average value during a solar cycle can validate a correlation and represent a corrective factor.

As shown in Fig. 4, where the trends of the Monthly Mean North Sunpsot Number [5] and the values of cps recorded at Jungfrauch NM64 (Jung1, Switzerland) and Kiel (Germany) stations during the $23^{\text {th }}$ and $24^{\text {th }}$ solar cycles are reported, there seems to be an inverse correlation between the two quantities. This confirms what indicated in introduction and that, at least at the high altitude of $3475 \mathrm{~m}$ a.s.l. of Jung1 station, the variation of the solar activity affects the value of the neutron flux rate with a percentage change of about $\pm 18 \%$ with respect the average cps value.
A similar comparison has been realized with the data provided by NMDB stations in Rome (Italy), Athens (Greece), Kiel (Germany), and others. The result of the comparison and the negligible difference between the measurements carried out in two different periods of the solar cycle suggest that the inverse correlation at ground level is weaker, such as demonstrated by the Kiel station measurement plot of Fig. 4 (altitude of the station $54 \mathrm{~m}$ a.s.l.).

Indeed, for stations placed at ground level it has been noted that, except for a few values that differ from an average trend, the percentage variations in neutron flux data during a solar cycle are on average very small, not exceeding about $6 \%$, i.e. of the same order of magnitude of the uncertainties of the measurements recorded by the ALNOR 2202D rem meter. This evaluation not significantly change if a variation range $\pm 2 \%$ due to the detection of proton component of the secondary cosmic radiation is included. This result, in some ways unexpected when compared with the variations of the Sunspot Number in a solar cycle (i.e. Fig. 4), leads to consider the variation of the neutron flux to be not significant, if referred to measurement uncertainties.

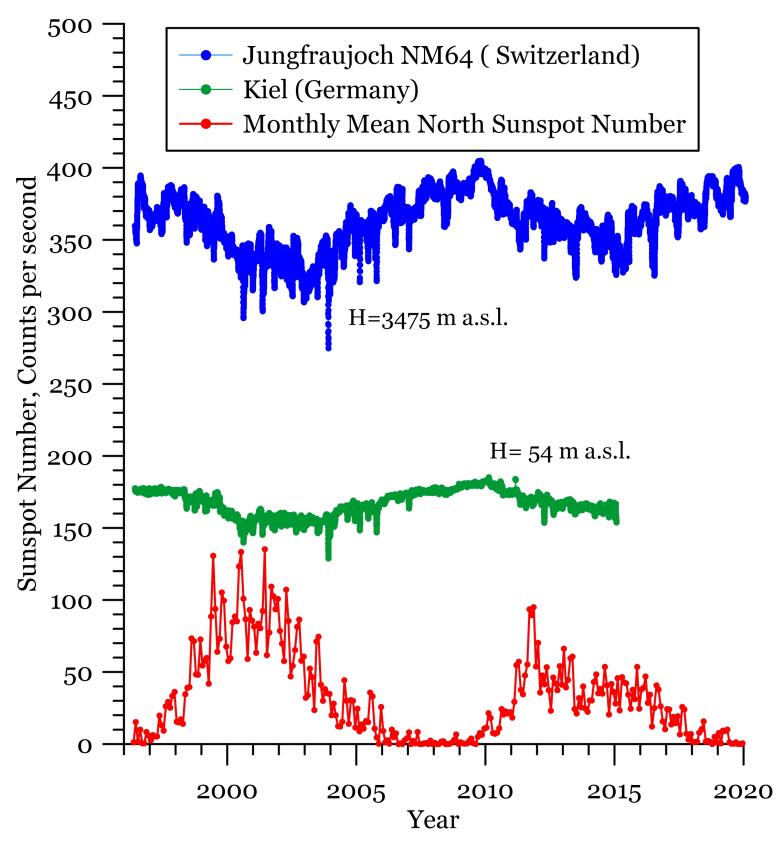

Figure 4. Comparison between the data measured at Jung1 and Kiel stations and Monthly Mean of North Hemispheric Sunspot Number during $23^{\text {th }}$ and $24^{\text {th }}$ solar cycle. For clarity of representation, both Jung1 and Kiel graphs are smoothed by calculating for each value a 9-point moving average.

For the aim of this work, the values of $\dot{H}^{*}(10)$ reported in Tab. 1 can be adopted for determining the irradiation time of electronic device to be tested without any correction, just taking into account the measurement uncertainties.

Therefore, with reference to the location of the measurement site, sensitivity of the instrumentation used, measurement precision, and so on, it is believed that a correction factor can be still applied if 
measurements are realized during periods of minimum or maximum of solar activity.

A reliable correlation with other environmental parameters is difficult to establish, for the small amount of data.

It seems that there is not significant difference between the measurements carried out with different temperature, pressure and humidity conditions while a very weak dependence on solar activity cannot be ruled out.

\section{CONCLUSIONS}

The aim of this work has been achieved with the evaluation of typical values of the neutron dose and flux rate at ground level, and correlating them with functioning parameters of electronic devices. The data obtained have been compared with those available in the literature, highlighting a good agreement among the results. The further comparisons with the data provided by the world neutron monitoring network allow to determine for a reference site an average value of the neutron "dose rate" at ground level, in terms of ambient dose equivalent rate, to which all devices are subjected.

The average variation of the neutron flux values at ground level over a solar cycle is very limited and of the same order of magnitude of measurement uncertainties. However, with reference to the sensitivity of the instrumentation and the desired precision of the measurements, it may still be useful to apply a correction factor that can be derived by comparing the measured and/or estimated values of solar activity with an average value of a reference neutron parameter.

Acknowledgements: The paper is a part of the research realized within the project RHAISP ( $R A D$ $H A R D$ AeroSpace Image Sensing and Processing) funded by Italian Foreign Office.

\section{REFERENCES}

1. F. Cannizzaro, G. Greco, M. Raneli, M. C. Spitale, E. Tomarchio, "Behaviour of $7 \mathrm{Be}$ Air Concentration observed during a period of 13 years and Comparison with Sun Activity," Nucl. Geophys., vol. 9, no. 6, pp. $597-607,1995$ DOI: 10.1016/0969-8086(95)00043-7

2. F. Cannizzaro, G. Greco, M. Raneli, M. C. Spitale, E. Tomarchio, "Concentration measurements of $7 \mathrm{Be}$ at ground level air at Palermo, Italy - Comparison with solar activity over a period of 21 years," J. Environ. Radioact., vol. 72, no. 3, pp. $259-271,2004$.

DOI: 10.1016/So265-931X(03)oo177-2

PMid: 14972409
3. S. Basile, R. Burlon, E. Tomarchio, "Analysis of $7 \mathrm{Be}$ and ${ }^{210} \mathrm{~Pb}$ concentration and $7 \mathrm{Be} / 210 \mathrm{~Pb}$ activity ratio in ground level air at Palermo (Italy)," Radiat. Eff. Defects Solids, vol. 174, no. $11-12$, pp. 998 - 1007, 2019 DOI: $10.1080 / 10420150.2019 .1683838$

4. Neutron Monitor DataBase (NMDB), European Commission, Brussels, Belgium.

Retrieved from: http://www01.nmdb.eu Retrieved on: Aug. 20, 2021

5. Sunspot Index and Long-term Solar Observations (SILSO), on-line Sunspot Number catalogue: years: 2012 and 2020, Royal Observatory of Belgium, Brussels, Belgium.

Retrieved from: http://www.sidc.be/silso/ Retrieved on: Aug. 20, 2021.

6. T. Nunomiya, S. Abe, N. Hirabayashi, T. Nakamura, "Sequential Measurements of Cosmic-Ray Neutron Energy Spectrum and Ambient Dose Equivalent on the Ground," J. Nucl. Sci. Technol., vol. 41, suppl. 4, pp. 466 - 469, Mar. 2004. DOI: $10.1080 / 00223131.2004 .10875748$

7. T. Nakamura et al., "Sequential Measurements of Cosmic-Ray Neutron Energy Spectrum and Ambient Dose Equivalent on the Ground," presented at the 11th Int. Conf. International Radiation Protection Association (IRPA 11), Madrid, Spain, May 2004.

8. T. Nakamura, T. Nunomiya, S. Abe, K. Terunuma, H. Suzuki, "Sequential Measurements of Cosmic-Ray Neutron Spectrum and Dose Rate at Sea Level in Sendai, Japan,” J. Nucl. Sci. Technol., vol. 42, no. 10, pp. $843-853$, Oct. 2005. DOI: $10.1080 / 18811248.2005 .9711035$

9. T. Nakamura, "Cosmic-ray Neutron Spectrometry and Dosimetry,” J. Nucl. Sci. Technol., vol. 45, suppl. 5, pp. 1 - 7, Jun. 2008. DOI: $10.1080 / 00223131.2008 .10875772$

10. M. Kowatari et al., "Evaluation of Geomagnetic Latitude Dependence of the Cosmic-ray Induced Environmental Neutrons in Japan," J. Nucl. Sci. Technol., vol. 44, no. 2, pp. $114-120,2007$. DOI: $10.1080 / 18811248.2007 .9711264$

11. K. Lee, R. Sheu, "Comparing two Measurements of the same Cosmic-ray neutron spectrum using standard Bonner Spheres and high-sensitivity Bonner cylinders," Radiat. Prot. Dosimetry, vol. 177, no. 4, pp. $450-457$, Dec. 2017 DOI: $10.1093 / \mathrm{rpd} / \mathrm{ncx} 063$ PMid: 29272885

12. D. A. H. Rasolonjatovo et al., "Measurement for the Dose-rates of the Cosmic-ray Components on the Ground," J. Radiat. Res., vol. 43, no. Suppl, pp. S27 - S33, Dec. 2002. DOI: $10.1269 /$ jrr.43.S27 PMid: 12793726

13. G. F. Krymsky, V. G. Grigor'ev, S. A. Starodubtsev, "New method for estimating the absolute flux and energy spectrum of solar cosmic rays based on neutronmonitor data," JETP Lett., vol. 88, no. 7, pp. $411-413$, Dec. 2008.

DOI: 10.1134/Soo21364008190016 